\title{
Automated Vehicle Behavior Design for Pedestrian Interactions at Unsignalized Crosswalks
}

\author{
$\underline{\text { Suresh Kumaar Jayaraman }}^{1}$, Lionel P. Robert Jr. ${ }^{2}$, Xi Jessie Yang ${ }^{3}$, and Dawn M. Tilbury ${ }^{1}$ \\ ${ }^{1}$ Department of Mechanical Engineering, University of Michigan, Ann Arbor, MI 48109, USA \\ ${ }^{2}$ School of Information, University of Michigan, Ann Arbor, MI 48109, USA \\ ${ }^{3}$ Department of Industrial and Operations Engineering, University of Michigan, Ann Arbor, MI 48109, USA
}

Keywords: autonomous urban driving, behavior-aware control, AV-pedestrian interaction

\section{Introduction}

A significant challenge for real-world deployment of automated vehicles (AVs) is their safe interaction with other road users, particularly pedestrians, whose behavior can change almost instantaneously $[1,2]$. These interactions, especially at uncontrolled mid-block crosswalks, are challenging as the right-of-way in the US varies by state [3]. To ensure safety, AVs are expected to drive cautiously around pedestrians. However, conservative AV behavior can encourage pedestrians to be careless [4]. Additionally, riders inside the AV expect a comfortable ride and to reach their destinations (AV performance) without excessive delay [5].

These objectives of safety, performance, and comfort are opposing in nature and thus have to be balanced in a way that is accepted by both the riders and the pedestrians. Existing approach of fixing the weights of the objectives $[6,7]$ can be constrictive as in reality, the objectives can vary depending on the situation. For example, in the case of pedestrian crossing at an unsignalized crosswalk, the AV might want to prioritize comfort normally and gradually slow down to stop, but at a school zone might want to prioritize safety and have a stronger deceleration. Further, existing approaches to AV decision-making around pedestrians consider pedestrians as independent moving obstacles $[8,7]$ and do not explicitly consider the effects of the vehicle behavior on the pedestrian behavior. Pedestrians, in general, perceive a defensive driving style (which prioritizes safety) as more acceptable and are more willing to cross under such situations [1,9]. However, the objective evaluation of these interactions - expressed in terms of distance gap accepted, average acceleration, average jerk (an indicator of sudden acceleration/deceleration), etc., of the different driving styles during pedestrian interaction are not clearly known.

The primary contribution of this research is the objective evaluation of a spectrum of driving behaviors represented by the different weight combinations of the three AV driving objectives - safety, performance, and comfort. We incorporated a hybrid systems pedestrian model that is interactive and depends on the vehicle behavior (distance and speed). Our findings suggest the possibility of characterizing driving behaviors by varying the weights of the driving objectives. We believe that by understanding the objective performance of the different driving behaviors with an interactive pedestrian model, the AV-decision making can be expressed by varying the weights of the objective function.

\section{Methodology}

We simulated a simple scenario with straight roads, similar to actual midblocks, and the AVs followed the center line of the lane as shown in Fig. 1. We assumed that AVs use only longitudinal control (acceleration/deceleration) 
while interacting with crossing pedestrians. Pedestrian crossing behavior was simulated using a data-driven hybrid systems model developed earlier using a virtual reality user study [10]. At any time, pedestrians could be doing one of four actions - approaching the crosswalk, waiting, crossing, and walking away from the crosswalk - which are taken as the discrete states of the pedestrian. We defined a finite state machine that had a set of possible transitions between the discrete states. The transitions could be triggered by pedestrian position or a pedestrian decision (to wait, to cross, to walk, which in turn depended on the AV's continuous actions). In addition, each of the discrete states had an associated continuous model that defined the evolution of the position and velocity for the pedestrian in that state, thus creating a hybrid systems model. The data for the pedestrian model was obtained through a user study [1], where pedestrians crossed the street with oncoming AVs in a simulated virtual reality environment as shown in Fig. 1. We assumed that pedestrian behavior depend on the instantaneous vehicle states.

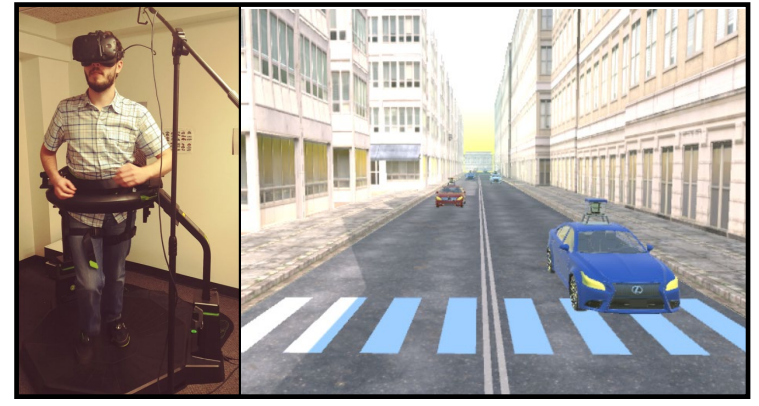

(a)

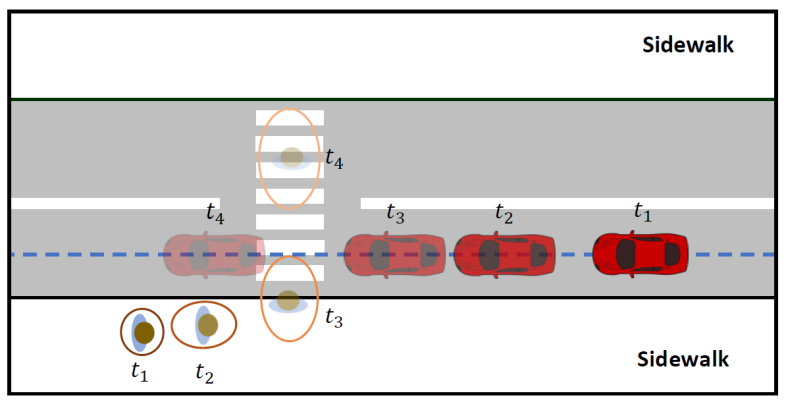

(b)

Figure 1: (a) Virtual reality experimental setup for user study (b) Representation of a typical interaction between a vehicle and a crossing pedestrian in the developed simulation; $t_{1}$ indicates the current time step states whereas $t_{2}, t_{3}$, and $t_{4}$ represent the predictions of pedestrian states and planned AV states.

We developed an MPC controller for the AVs that used predictions of the pedestrian behavior. We assumed the AV to be a point mass with a rectangular footprint and employed a discrete longitudinal kinematic model shown in equation (1), where $x, v, a$ are the longitudinal position, speed, and acceleration inputs of the vehicle respectively. $\Delta t$ denotes the discretization time step.

$$
\left[\begin{array}{l}
x_{k+1} \\
v_{k+1}
\end{array}\right]=\left[\begin{array}{l}
x_{k} \\
v_{k}
\end{array}\right]+\Delta\left[\begin{array}{l}
v_{k} \\
a_{k}
\end{array}\right]
$$

\subsection{Cost Function and Constraints}

Safety is a main priority in the AV planning and control problem. However, the AVs should also adhere to the speed limits and reach their destination quickly while maintaining ride comfort for the people inside. The optimization objective is formulated as the minimization of a cost function. This cost function serves as an inverse description of the ideal vehicle behavior and consists of three types of costs - safety, performance, and comfort. The safety cost is a function of the distance of the AV from the pedestrian when the pedestrian is in the same lane as the AV. The performance cost is given by the distance to the target location and the deviation of the AV speed from the speed limit. The comfort cost is given by the magnitude of acceleration and jerk. The cost function is thus represented by,

$$
\begin{aligned}
J & =J_{\text {safety }}+J_{\text {performance }}+J_{\text {comfort }} \\
J_{\text {safety }} & =w_{s} f_{s}\left(x_{\text {Ped }}, x\right) \\
J_{\text {performance }} & =w_{p}\left(f_{t}\left(x, x_{\text {ref }}\right)+f_{v}\left(v, v_{\text {ref }}\right)\right) \\
J_{\text {comfort }} & =w_{c} f_{c}(a, \Delta a)
\end{aligned}
$$


AV motion is constrained to follow the model discussed in equation (1). States and inputs are also constrained considering the physical limitations of the vehicle. Although the inclusion of the safety cost could result in safe vehicle maneuvers, minimal safety of collision avoidance is enforced by incorporating collision avoidance constraints in addition to the safety cost, similar to [11]. Pedestrian trajectory was predicted using the hybrid systems model for a horizon of 3 seconds.

\subsection{Implementation}

We developed the simulation to be as realistic as possible by considering a stream of AVs approaching the crosswalk one after the other with varying speeds and time gaps. However, at any time only one pedestrian was in the simulation. We assumed that all pedestrians had the intention to cross the street. AVs spawned with a random initial speed $(13-16 \mathrm{~m} / \mathrm{s})$ and a randomly varying time gap $(1-7 \mathrm{~s})$ between their spawns. The different cost functions have scaling factors to ensure similar ranges of the absolute values for $f_{s}, f_{c}$, and $f_{t}+f_{v}$. This ensures that the safety, performance, and comfort costs are similar under same weights. We evaluated seven combinations of the weights as shown in Table 1. Simulations were run 200 times for each of the seven weight combinations. The performance weight was ensured to be non-zero so that the vehicle moves forward, especially after stopping for a crossing pedestrian.

Table 1: Different combinations of the three weight parameters.

\begin{tabular}{llll}
\hline \hline Weight combination & $w_{s}$ & $w_{p}$ & $w_{c}$ \\
\hline S & 0.950 & 0.050 & 0.000 \\
P & 0.000 & 1.000 & 0.000 \\
C & 0.000 & 0.050 & 0.950 \\
PC & 0.000 & 0.500 & 0.500 \\
SC & 0.475 & 0.050 & 0.475 \\
SP & 0.500 & 0.500 & 0.000 \\
SPC & 0.333 & 0.333 & 0.333 \\
\hline
\end{tabular}

\section{Results}

The objective evaluation of the various controllers with the different weight combinations is shown in Table 2 . Collisions were observed in rare situations even with the presence of collision avoidance constraints due to the physical limitations of the vehicle. It can be observed that the inclusion of the safety cost in any combination increases the safety metrics as observed through the increased distance to pedestrians $\left(d_{p e d}\right)$, and reduced probability of collision $\left(p_{c o l}\right.$. Incorporating a safety cost in addition to the collision avoidance constraints helps the vehicle in slowing down earlier as expressed by the lower average velocity $\left(a_{m}\right)$ and higher interaction time $\left(t_{\text {int }}\right)$.

The inclusion of the comfort cost improved the comfort metrics as expected, by having a lower absolute jerk $\left(j_{m}\right)$ and acceleration values $\left(a_{m}\right)$. Similarly, the inclusion of the performance cost reduces the time to destination $\left(t_{d e s}\right)$, but increases the chances of collision $\left(p_{c o l}\right)$. An interesting observation is that the inclusion of performance cost, sometimes results in the vehicle not yielding to the pedestrians expressed by $p_{n y}$. A reason for this could be that the AV velocity is higher and the pedestrian is close to the AV such that it is safer to accelerate and pass the crosswalk than to decelerate and try to stop. This suggests a possibility of expressing AV decisions to yield/not yield by suitably varying the weights.

\section{Conclusion}

In our research we explored how the different vehicle objectives affect the vehicle performance during interaction with pedestrians. We developed a simulation environment based on a data-driven hybrid systems pedestrian model and MPC controllers for AVs, which provides a platform for future testing. We found that a safety cost in addition to collision avoidance constraints greatly improves the safety performance metrics. Our findings suggest that AV decision-making can potentially be expressed by adjusting the weights of the different costs. 
Table 2: Comparison of average performance metrics for pedestrians for the various weight combinations.

\begin{tabular}{|c|c|c|c|c|c|c|c|}
\hline Metric & $\mathrm{S}$ & $\mathbf{P}$ & $\mathbf{C}$ & PC & $\mathrm{SC}$ & SP & SPC \\
\hline$t_{\text {int }}[s]$ & 7.54 & 5.38 & 6.08 & 6.09 & 7.21 & 7.18 & 7.01 \\
\hline$t_{\text {des }}[s]$ & 9.75 & 7.46 & 9.47 & 8.73 & 8.78 & 9.08 & 8.91 \\
\hline$d_{\text {ped }}[m]$ & 7.34 & 4.33 & 5.20 & 5.85 & 6.38 & 6.48 & 6.21 \\
\hline$v_{m}[\mathrm{~m} / \mathrm{s}]$ & 12.10 & 13.80 & 13.30 & 13.19 & 12.38 & 12.08 & 12.20 \\
\hline$a_{m}\left[\mathrm{~m} / \mathrm{s}^{2}\right]$ & -0.15 & -0.20 & -0.06 & -0.19 & -0.14 & -0.11 & -0.12 \\
\hline$j_{m}\left[m / s^{3}\right]$ & 0.08 & 0.10 & 0.04 & 0.08 & 0.07 & 0.12 & 0.09 \\
\hline$p_{\text {col }}$ & 0.00 & 0.01 & 0.00 & 0.01 & 0.00 & 0.00 & 0.00 \\
\hline$p_{n y}$ & 0.00 & 0.05 & 0.02 & 0.03 & 0.00 & 0.00 & 0.00 \\
\hline
\end{tabular}

$t_{\text {int }}$ - interaction duration, time when AVs first predicted pedestrians to step into the lane to the time

when the pedestrians step out of the lane or when AVs crossed the pedestrian, whichever is earlier

$t_{\text {des }}$ - time for the AV to reach the destination

$d_{\text {ped }}$ - minimum distance to pedestrian when pedestrian is crossing

$v_{m}$ - average velocity during interaction defined by $t_{\text {int }}$

$a_{m}$ - average acceleration during interaction

$j_{m}$ - average absolute jerk during interaction

$p_{\text {col }}$ - probability of collision given by the ratio of the number of collisions observed to the total

number of simulation run for that condition

$p_{n y}$ - probability of not yielding to the pedestrians given by the number of runs the vehicle did not

yield to the total number of simulation run for that condition

\section{References}

[1] S. K. Jayaraman, C. Creech, D. M. Tilbury, X. J. Yang, A. K. Pradhan, K. M. Tsui, and L. P. Robert, "Pedestrian trust in automated vehicles: Role of traffic signal and av driving behavior," Frontiers in Robotics and AI, vol. 6, p. 117, 2019.

[2] L. Robert, "The future of pedestrian-automated vehicle interactions," XRDS: Crossroads, ACM, vol. 25, no. 3, 2019 .

[3] D. Shinkle, "Pedestrian crossing: 50 state summary," Proc. Nat. Conf. State Legislatures, 2016.

[4] A. Millard-Ball, "Pedestrians, Autonomous Vehicles, and Cities," J. Planning Edu. Res., vol. 38, pp. 6-12, 2018.

[5] R. Krueger, T. H. Rashidi, and J. M. Rose, "Preferences for shared autonomous vehicles," Transportation research part C: emerging technologies, vol. 69, pp. 343-355, 2016.

[6] M. Kuderer, S. Gulati, and W. Burgard, "Learning driving styles for autonomous vehicles from demonstration," in 2015 IEEE International Conference on Robotics and Automation (ICRA), pp. 2641-2646, IEEE, 2015.

[7] I. Batkovic, M. Zanon, M. Ali, and P. Falcone, "Real-time constrained trajectory planning and vehicle control for proactive autonomous driving with road users," arXiv preprint arXiv:1903.07743, 2019.

[8] H. Bai, S. Cai, N. Ye, D. Hsu, and W. S. Lee, "Intention-aware online POMDP planning for autonomous driving in a crowd," in IEEE Int. Conf. Robot. Autom., pp. 454-460, IEEE, 2015.

[9] R. Zimmermann and R. Wettach, "First step into visceral interaction with autonomous vehicles," in Proceedings of the 9th International Conference on Automotive User Interfaces and Interactive Vehicular Applications, pp. 58-64, New York,NY: ACM, 2017.

[10] S. K. Jayaraman, D. Tilbury, X. J. Yang, A. K. Pradhan, and L. P. Robert Jr., "Analysis and prediction of pedestrian crosswalk behavior during automated vehicle interactions," Manuscript under review, 2019. Paper draft available in https://umich.box.com/s/u7x3pcf3jhd0potl10z6ya5dy7ofy457.

[11] S. Bertrand, J. Marzat, H. Piet-Lahanier, A. Kahn, and Y. Rochefort, "Mpc strategies for cooperative guidance of autonomous vehicles," 2014. 